\title{
Stakeholders' views on the routine use of $n$-of- 1 trials to improve clinical care and to make resource allocation decisions for drug use
}

\author{
Jane Nikles ${ }^{1,4}$ MBBS, PhD, Post-doctoral Research Fellow, School of Medicine \\ Geoffrey K. Mitchell ${ }^{1}$ PhD, RACGP, Professor, PHCRED Senior Career Research Fellow, \\ School of Medicine, Discipline of General Practice \\ Alexandra Clavarino ${ }^{1} \mathrm{PhD}$, BSW, Senior Lecturer, School of Pharmacy \\ Michael J. Yelland ${ }^{2}$ PhD, FRACGP, Associate Professor in Primary Health Care \\ Christopher B. Del Mar ${ }^{3}$ MD, FRACGP, Pro-Vice Chancellor (Research); and Dean, \\ Health Sciences and Medicine \\ ${ }^{1}$ School of Medicine, The University of Queensland, Brisbane, QLD 4006, Australia. \\ ${ }^{2}$ School of Medicine, Griffith University, Brisbane, QLD 4131, Australia. \\ ${ }^{3}$ Faculty of Health Sciences and Medicine, Bond University, Gold Coast, QLD 4229, Australia. \\ ${ }^{4}$ Corresponding author. Email: uqjnikle@uq.edu.au
}

\begin{abstract}
N-of-1 trials are empirical formal tests using a within-patient randomised, double-blind, cross-over comparison of drug and placebo (or another drug), which we adapted to study individual patients' responses as a clinical tool to guide clinical management. We administered semi-structured interviews to gauge stakeholder perspectives on the possibility of using routine n-of-1 trials for this purpose. Stakeholders included government and non-government health care sector, and patient, clinician and consumer, organisations. Stakeholders supported more widespread implementation of n-of- 1 trials, in a targeted fashion, with some caveats. Barriers to their widespread implementation included constraints on doctors' time, doctors' acceptance, drug company acceptance, patient willingness, and cost. Strategies for overcoming barriers included conditional Pharmaceutical Benefits Scheme listing if cost-effective. There was little consensus on which model of n-of-1 trial implementation would be most effective. We discuss different approaches to addressing the several concerns raised to enable widespread introduction of $\mathrm{n}$-of-1 trials into routine clinical practice as a decision tool.
\end{abstract}

What is known about the topic? No previous publications have explored methods of implementation and gathered stakeholder's views on widespread use of n-of-1 trials.

What does this paper add? N-of-1 trials could have a role in making resource allocation decisions for medications, and in guiding treatment choices for patients.

What are the implications for practitioners? There is potential for n-of- 1 trials to be used more widely, including as part of normal medical practice for targeted illnesses and groups of patients. This could deliver clinical and economic benefits throughout the health care system.

Australian Pharmaceutical Benefits Scheme (PBS) and Repatriation PBS expenditure is over AU\$4 billion per year. A rational approach to reducing this cost is to target expensive drugs only to patients whose disease responds. Clinicians often informally test this (as empirical 'trials of treatment') in clinical practice. However, this has several potential biases which would be addressed by testing through n-of-1 trials. These are empirical tests using a within-patient randomised, double-blind, cross-over comparison of drug and placebo (or another drug), principally to study individual patients' responses where there is uncertainty about the effectiveness of a medication for a chronic condition.
In 1998, the Centre for General Practice at The University of Queensland established a national coordinating centre providing $\mathrm{n}$-of- 1 trials by mail and telephone to clinicians Australia-wide. $^{1-6}$ In patients where there was uncertainty about medication effectiveness, the results showed that of cases tested:

- in attention-deficit hyperactivity disorder, stimulants were superior to placebo in $42 \%^{2}$;

- in osteoarthritis, ibuprofen was superior to paracetamol in $17 \%$ of cases $^{3}$ and celecoxib was superior to paracetamol in $25 \%^{4}$; and 
- in chronic neuropathic pain, gabapentin was superior to placebo in $29 \%{ }^{7}$

$\mathrm{N}$-of-1 trials have the potential to facilitate long-term concordance with medications if these have been proven to be beneficial for the individual. ${ }^{6}$ With equity of access Australia-wide ensured by utilising post and telephone delivery (important in rural and remote areas), n-of-1 trials have the potential to improve health outcomes for chronic conditions. This could be achieved by (1) increasing rational and cost-effective prescribing; and (2) improving patient outcomes-to-adverse-events ratios. ${ }^{1}$

At present, n-of-1 trials are not widely used. Although the technique could become part of normal medical practice for targeted illnesses and groups of patients, it is resource intensive because of the need for special medication packaging, record keeping and so on, and cost-effectiveness varies across drugs and patients. ${ }^{1,2}$

We endeavoured to obtain feedback from various stakeholder groups about the feasibility and usefulness of a widespread implementation of n-of-1 trials in clinical practice.

\section{Methods}

Sample

A list of key stakeholders (government and non-government health care sector organisations and patient and clinician and consumer organisations) was compiled. Stakeholders had varying levels of familiarity with n-of- 1 trials. We approached every organisation we were aware of which had, or was likely to have, an interest in the broader application of n-of- 1 trials or play a role in whether $n$-of- 1 trials would be offered on a broader scale than they currently are. A sample was purposively selected from different categories of respondents (e.g. consumers, doctors, government, industry) to provide an optimally broad range of responses. $^{8}$

Of the twelve interviewees, five were located in the Australian Capital Territory, three in New South Wales, two in Queensland and two in Victoria. Interviewees included several representatives of Medicare Australia and the Therapeutic Goods Administration, two representatives of the Royal Australian College of General Practitioners (RACGP), one consumer representative, one drug company director, two members of quality use of medicines advocacy groups, and two pharmacists who had been involved in conducting n-of-1 trials.

\section{Data collection}

Stakeholders were briefed about the project and then sent a list of questions (developed and piloted by the co-investigators) to consider before the interview. The questions were open-ended and were explored within semi-structured interviews. Questions were adapted for each target group - different questions were used for consumers, medical practitioners, government and private industry.

Two interviewers ( $\mathrm{J}$ N and R C) separately carried out all the interviews. From April to November 2005 we interviewed participants by telephone until no new data were obtained. Ten interviews were conducted with 12 interviewees ( 2 interviews were with 2 interviewees). All interviews were $\sim 30$ minutes in length. Interviews were taped and transcribed by the interviewer.

\section{Data analysis}

Data were analysed by visual inspection, sentence-by-sentence, generating themes. A thematic coding scheme was then developed. After the text was organised into these themes, core themes were identified and subcategories were created, and linked together. Finally, core themes were integrated to form a story. The process was repeated until no further themes emerged. All reasonable steps have been taken to maintain participant confidentiality. The study was approved by the University of Queensland Medical Research Ethics Committee.

\section{Results}

The comments made by the participants in relation to each question are presented below.

\section{The rationale for $n$-of- 1 trials}

All participants seemed to have a good understanding of what an n-of- 1 trial was and the rationale behind its use. Rationales that were given included:

- Increasing cost-effectiveness of government spending and improving community health outcomes.

- Maximising effectiveness of patient/drug combination, assuring patient response matches prescription intention.

\section{Benefits of n-of- 1 trials}

All participants seemed to have a clear understanding of the potential benefits of $n$-of- 1 trials. Targeting of therapy, reducing unnecessary prescribing, and reduction in health care costs were some of the main benefits mentioned.

\section{Barriers to widespread use of n-of- 1 trials}

The substantial cost of provision of n-of- 1 trial services was a concern for several respondents, and it was felt that this would need to be addressed early in a strategy for widespread provision of n-of-1 trial services.

There was some concern about the acceptability of the n-of-1 trials, particularly with regard to how well the clinical model of GPs conducting n-of-1 trials fits with normal general practice, in which formal testing is not usual. Moreover, n-of- 1 trials require a paradigm shift for doctors, away from standard randomised controlled trials (RCTs), to focusing on drug effectiveness in the individual patient. From the patient point of view, n-of-1 trials require substantial commitment and can be time consuming, especially the regular completion of diaries. Patient willingness might depend on drug access issues via the Pharmaceutical Benefits Scheme (PBS), side-effects and length of the n-of-1 trial.

Ethical and regulatory issues also arose - some respondents felt it was unethical if an effective drug was not being offered (i.e. only placebo was being offered) at a particular time. Ethical issues also arose around blinding of treatment: should patients know what they are taking?

It was questioned whether n-of-1 trials should be a condition of listing a drug on the PBS, allowing patient reimbursement. It was felt that if there was a response that was too small to be 
significant, it would be unethical to withdraw the subsidy, and this would require ethical clearance.

\section{Key information needed to consider n-of-1 trials feasible}

GP-based informants considered n-of-1 trials would be feasible if there were payments for nurse practitioners, who could provide appropriate support for clinicians; if PIP (practice incentive payments for GPs) or other funding avenues were available for resourcing n-of-1 trials; and if a suitable business case was constructed.

The greatest efficiencies will occur with the treatment of long-term, chronic and stable conditions, with high-cost drugs. This suggests that a targeted application of n-of- 1 trials should be recommended. For example, they could have a place where variation in response cannot be reasonably predicted by clinical or test measurements. (GP organisation representative.)

Incorporation of n-of-1 trials into existing models of GP consultations - for example, if they became part of standard procedure for doctors when considering the use of certain drugs would enhance their feasibility. It was clear from participant responses that n-of-1 trials would only be feasible for certain categories of patients, certain drugs and certain clinical indications. Also, participants were concerned about how privacy issues would be dealt with.

Factors influencing acceptance and uptake of n-of-1 trials by consumers, government, industry, doctors and pharmacies

\section{Consumers}

One respondent felt that n-of-1 trials focus on the complaint and give the client an opportunity to 'do something'. There was a consensus that n-of- 1 trials need to be carefully promoted to consumers - especially if the n-of-1 trial raised a concern that was not there already - and there needs to be a well thought out communication strategy. The take-home message from consumers was that 'n-of-1 trials need to be suitable for real world conditions'. (Consumer representative.) For example, patients would need to be reliably informed of the importance of completing trial documentation.

\section{Government}

All respondents agreed that for n-of- 1 trials to be effective there must be a positive impact on policy. There was agreement that n-of-1 trials are suitable for expensive drugs used in small numbers of people. Participants also felt there was a need to consider who would be providing the service, and whether there would be a need for certification. Target groups and sub-groups for particular drugs would need to be defined. Issues mentioned included political considerations (for example, that the implementation of n-of-1 trials must not worsen the government's political position) and workforce considerations (that n-of-1 trials may increase the need for doctors).

Financial considerations identified included funding for trials - specifically, who would fund the program and how much impact each stakeholder's involvement would have on their internal costings. One respondent thought that although patients may reduce their medication, the PBS may not fund drugs based only on an n-of-1 trial - n-of-1 trials would be additional to the usual information, not stand-alone. Also, there is a cut point in terms of the time the n-of- 1 trial runs for before recouping the costs. However, cost-effectiveness should not be a prime driver; more individual patient management is a more important consideration. Another respondent commented that

Payers and sellers have an interest in situations where there is a product with uncertain cost-effectiveness such as in the case of a drug with a troublesome history of PBAC [Pharmaceutical Benefits Advisory Committee] application(s). Where there are data to support increased cost-effectiveness under an n-of- 1 trials regime, stipulating the requirement for $n-o f-1$ trials for a couple of years may be warranted. (Therapeutic Goods Administration representative.)

Criteria suggested by participants in relation to choosing which drugs to conduct n-of-1 trials for were 'Is the drug suitable for n-of-1 trials?'; 'Are there uncertainties of clinical and cost-effectiveness?' and 'Is the n-of-1 trial going to answer the clinical question?' In relation to using the n-of-1 trial option as a tool for PBS listing, comments included that if the number of suitable drugs were too small, it would not be successful; and if a random and representative sample of all patients could be obtained, it would be feasible to conduct an n-of- 1 trial only in sample patients.

\section{Industry}

There was qualified support from industry for n-of-1 trials to be included as part of the PBAC application process, particularly where the incorporation of n-of-1 trial conditions might facilitate the listing of some drugs not otherwise conforming to usual cost-effectiveness criteria. It was noted that the pharmaceutical industry may have some vested interests, especially where PBS listing might be concerned.

\section{Doctors}

Doctors are key players in the adoption of routine n-of-1 trials. As one participant said:

If n-of- 1 trials became part of the PBS then GPs will respond but may not like it. Political and financial acceptability is needed for quick uptake but clinical acceptance is essential for a program to be successful. (Quality use of medicines advocacy group representative.)

It was clear that the main issue would be principally about time - time to 'sell' the process to the patient, and time to administer the process, and make appropriate communications. Doctors would need to have the capacity to organise the trials within their practice and to be convinced that the benefits were worthwhile. They would also need to assess the patient's capacity to see the process through. It was queried whether the n-of-1 trial requires the doctor to do work outside of the consultation, and if so it was suggested that there would need to be a Medicare Benefits Scheme (MBS) item number to cover the overheads. 
It was felt that early adopters, attracted by the possibility that n-of-1 trials could decrease the uncertainties of general practice, would take on n-of-1 trials, but that uptake by other clinicians would be slow. Incentives for doctors would be needed, but 'if payment is linked to anything, people complain about red tape'. It might be better for n-of- 1 trials to be sponsored through alternative government funding avenues such as a teaching or quality assurance and continuing professional development (QA\&CPD) approach rather than the MBS model. In these cases, the RACGP would need to be involved.

A number of practical issues were raised, such as integration with medical desktop software (e.g. Medical Director), and building systems for support and financial compensation within general practices (e.g. for nurse and other staff time). The whole process would need to be managed through a trusted agency/source, with credentials in clinical excellence (e.g. the RACGP [perhaps with QA\&CPD involvement], Cochrane centres, the National Prescribing Service [NPS - perhaps with involvement of Divisional NPS coordinators] or the Drug and Therapeutics Information Service [DATIS]).

Questions were raised about how n-of-1 trials would work with patients on multiple drugs, whether they could be applicable for drugs used for their registered indications and drugs used outside these indications, and how they might affect medical defence (e.g. whether they would provide evidence of inappropriate use of drugs). Other concerns were related to implications for a new edition of standards for general practice, implications in relation to the Privacy Act, particularly where data are sent to external agencies, and ethical issues, including obtaining informed consent, especially where blinding to treatment is concerned.

\section{Hospital pharmacy}

One of the participants commented that pharmacies need to consider practical issues such as outsourcing of drug packaging. She felt that n-of- 1 trials could be feasible for hospital pharmacies:

Involvement in an n-of-1 trial would be feasible if the pharmacy is not setting it up and someone else is analysing the data. N-of-1 trials have longterm financial implications - the hospital will save money if patients do not need the drug. Providing a national service with one party doing all the data analysis would be feasible. (Hospital pharmacist.)

\section{Drugs for which routine n-of-1 trials may be useful}

Most stakeholders mentioned categories of drug rather than specific drugs. Chronic stable conditions and drugs with a short onset of action for both active and comparator were assumed. Suggestions included:

- drugs with therapeutic and cost-effectiveness dilemmas;

- the condition needs to be symptomatic or have a good surrogate marker;

- some highly specialised high-cost drugs with weak or no clinical trial data; and
- drugs where modified dose regimes are efficacious, such as programmed-release drugs where the relative efficacy of dose variations is difficult to demonstrate by RCT.

\section{Commercialisation of $n$-of- 1 trials}

There were no overtly negative opinions expressed regarding commercialisation, but most participants appeared guarded about this idea. Suggestions for increasing the feasibility of commercialisation included being useful to the PBS, conditional PBS listing, MBS reimbursement, provision by an independent, government-sponsored organisation, with GP incentives, and n-of-1 trials being highly targeted.

\section{Provision of resources for $n$-of- 1 trial services}

Stakeholders were unanimous in suggesting that drug providers'/ vendors' and governments' public health budgets provide the necessary resources, the latter either via the MBS, or (perhaps preferentially) through the Divisions of General Practice, with PIPs being the key resource. Another source of funds in the case of certain drugs, for example for gabapentin, could be the hospital sector.

\section{Models for a general uptake of n-of-1 trials}

It was suggested that a 'matching the model to the drug' approach would be more reasonable than a 'one model fits all drugs' approach. Pekarsky has suggested that there seem to be three key elements to an n-of-1 trial implementation model.

- Will n-of-1 trials be funded by PBS or hospitals for a specific drug?

- Should the drug be funded for a given patient?

- When is the prescriber required to offer an n-of-1 trial to a patient?

She set out seven model options (see Box 1) which were given to participants for comment.

There was no consensus on which models would be most effective. Some said it was not possible to generalise, because the model would have to work for the particular drug under consideration.

\section{Discussion}

N-of-1 trials have been used as a research method without breaking into clinical territory, where their potential to help make rational decisions about individual patient treatment choices remains largely unrealised. However, some recent publications from overseas show that there is ongoing momentum to bring n-of-1 trials, which offer a unique opportunity to individualise patient care, into their niches in mainstream medicine. Examples of several niches for n-of-1 trials include: in the assessment of regulatory approval for new treatments for rare and metabolic diseases ${ }^{9}$; in palliative care ${ }^{10}$; in traumatic brain injury in children (unpubl. data); and in developing policies for use of expensive agents, ${ }^{1}$ (for example, in the treatment of rheumatoid arthritis in the United States, where offering patients with rheumatoid arthritis an n-of-1 trial to compare methotrexate with etanercept could save costs relative to open access while preserving clinical freedom relative to mandatory stepped care $^{11}$ ). 


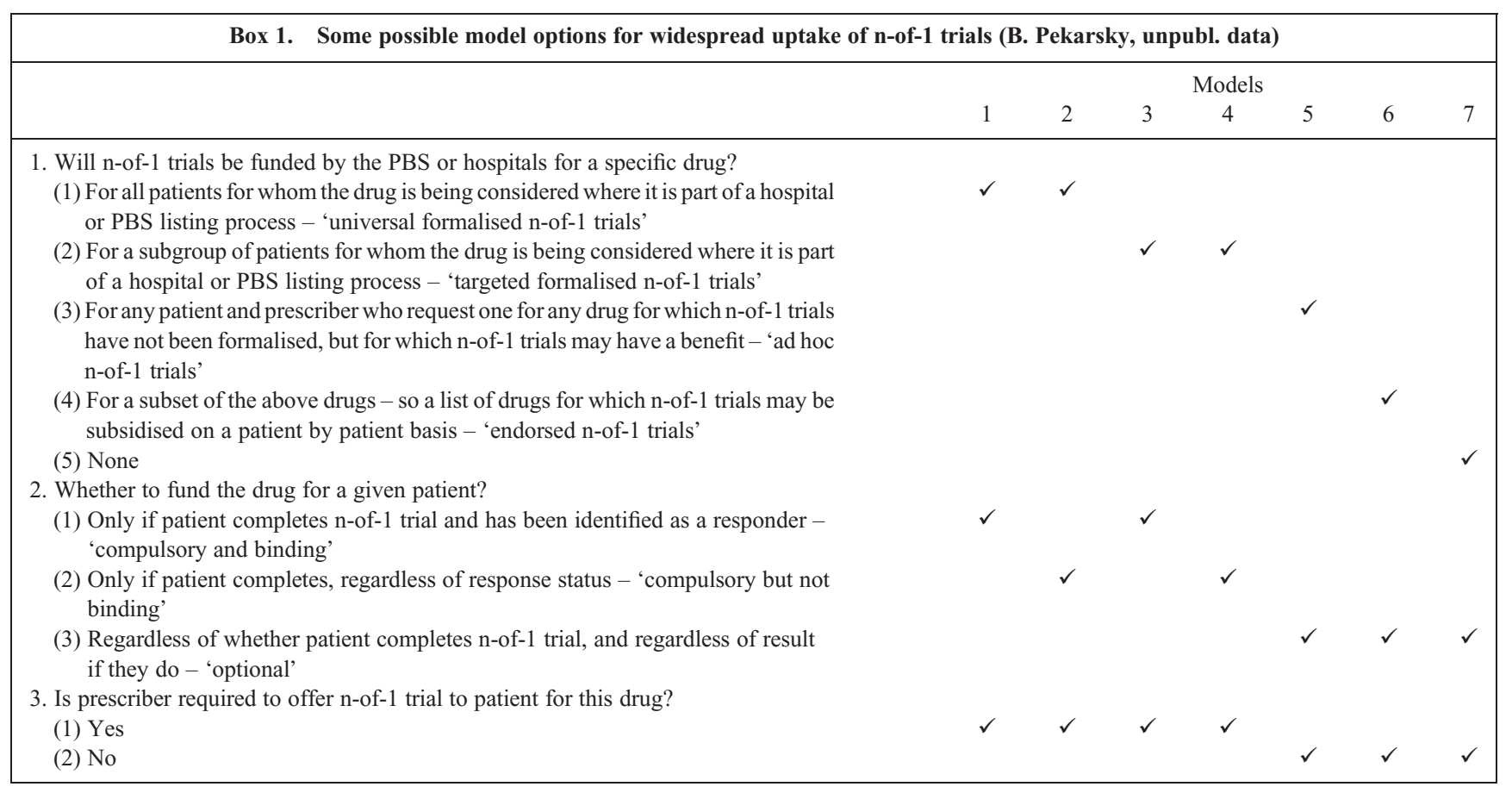

A recent paper outlines the history of and the barriers to use of n-of-1 trial services. Kravitz comments that limited evidence suggests that patients may be receptive to n-of-1 trials once they understand the benefits. ${ }^{12}$ Two pilot studies examining patient perspectives suggest that patients do perceive n-of- 1 trials to be an acceptable approach to the individualisation of treatment. ${ }^{5,13}$ Indeed, a patient-focused approach to the roll-out of any widespread use of n-of-1 trials is likely to be essential.

The widespread use of n-of- 1 trials would throw up technical methodological questions. The single-case experimental design scale aims to provide a brief and valid evaluation of methodological quality of single-subject (or n-of-1) designs. Items from the scale can be used as a checklist in the design, reporting and critical appraisal of single-subject design, thereby helping to improve standards of single-case methodology. ${ }^{14}$ A series of guidelines for reporting on n-of-1 trials similar to the CONSORT guidelines for RCTs is currently being developed. The ethics of routine n-of-1 trials are under discussion. ${ }^{15}$

Our data provide evidence about how n-of-1 trials might play a possible role and how they might work logistically within Australia. Perhaps this would include introducing a new MBS item number. Alternatively, n-of-1 trials could be sponsored through another government funding avenue (such as a teaching or QA\&CPD approach involving the RACGP). The whole process might be best managed through a trusted agency/organisation, with credentials in clinical excellence. Even the Pharmaceutical Benefits Subsidy Scheme could fund n-of-1 trials of interest in deciding the usefulness of specific expensive drugs. Widespread provision of n-of- 1 trials would be complex to implement, but with a carefully planned strategic approach has the potential to benefit not only individual patients, but also the Australian health care system.
Some barriers to widespread implementation include constraints on doctors' time, doctors' acceptance, drug company acceptance, patient willingness, and cost. Some strategies for overcoming these barriers include 1) building n-of-1 trials into the standard doctor consultation, 2) a carefully thought out social marketing approach, 3) providing incentives, for example through PIPs, or other avenues, such as payments for nurse practitioners who could provide appropriate support for doctors, 4) making sure that n-of-1 trials are suitable for real-world conditions and free of charge for patients, 5) keeping n-of- 1 trials no longer than 12 weeks in total, and 6) obtaining drug provider/vendor and government funding for an independent organisation to conduct n-of-1 trials.

Our study has some limitations. For example, although purposive sampling is appropriate for helping to understand the breadth of opinions, it cannot inform the relative importance of individual opinions. Note, though, that key stakeholders with a potential interest in n-of-1 trials were identified and participated in this study. Nevertheless, the study enables debate which could lead to more widespread implementation of n-of- 1 trials.

This study represents an important preliminary stage in gathering information about the process of more widespread implementation of n-of-1 trials, and is also a springboard for developing a broader picture incorporating more diverse perspectives. This would be necessary if n-of- 1 trials were to become more broadly used, so that they could realise their significant potential both to individualise patient care and to efficiently gather evidence equivalent in rigour to RCTs within the applications for which they are suitable.

\section{Competing interests}

The authors declare that they have no competing interests. 


\section{Acknowledgement}

We thank the Australian Health Ministers' Advisory Council for funding; Richard Clark and Brita Pekarsky for their contributions in the early stages of the project; Richard Clark for conducting some interviews; and Brita Pekarsky for contributing the model shown in the Box.

\section{References}

1 Scuffham PA, Yelland MJ, Nikles J, Pietrzak E, Wilkinson D. Are N-of-1 trials an economically viable option to improve access to selected high cost medications? The Australian experience. Value Health 2008; 11(1): 97-109. doi:10.1111/j.1524-4733.2007.00218.x

2 Nikles CJ, Mitchell GK, Del Mar CB, Clavarino A, McNairn N. An n-of-1 trial service in clinical practice: testing the effectiveness of stimulants for attention-deficit/hyperactivity disorder. Pediatrics 2006; 117(6): 2040-6. doi:10.1542/peds.2005-1328

3 Nikles CJ, Yelland M, Glasziou PP, Del Mar C. Do individualised medication effectiveness tests (n-of-1 trials) change clinical decisions about which drug to use for osteoarthritis and chronic pain? Am J Ther 2005; 12(1): 92-7. doi:10.1097/00045391-200501000-00012

4 Yelland MJ, Nikles CJ, McNairn N, Del Mar CB, Schluter PJ, Brown RN. Celecoxib compared with sustained-release paracetamol for osteoarthritis: a series of n-of-1 trials. Rheumatology 2007; 46(1): 135-40. doi:10.1093/rheumatology/kel195

5 Nikles CJ, Clavarino AM, Del Mar CB. Using n-of-1 trials as a clinical tool to improve prescribing. Br J Gen Pract 2005; 55(512): 175-80.

6 Nikles CJ, Mitchell GK, Del Mar CB, McNairn N, Clavarino A. Longterm changes in management following n-of- 1 trials of stimulants in attention-deficit/hyperactivity disorder. Eur J Clin Pharmacol 2007; 63(11): 985-9. doi:10.1007/s00228-007-0361-x

7 Yelland MJ, Poulos CJ, Pillans PI, Bashford GM, Nikles CJ, Sturtevant JM, et al. N-of-1 randomized trials to assess the efficacy of gabapentin for chronic neuropathic pain. Pain Med 2009; 10(4): 754-61. doi:10.1111/j.1526-4637.2009.00615.x
8 Pope C, Mays N. Reaching the parts other methods cannot reach: an introduction to qualitative methods in health and health services research. BMJ 1995; 311: 42-5.

9 Griggs RC, Batshaw M, Dunkle M, Gopal-Srivastava R, Kaye E, Krischer J, Nguyen T, Paulus K, Merkel PA. Rare Diseases Clinical Research Network. Clinical research for rare disease: opportunities, challenges, and solutions. Mol Genet Metab 2009; 96(1): 20-6. doi:10.1016/j.ymgme.2008.10.003

10 Keeley PW. Improving the evidence base in palliative medicine: a moral imperative. J Med Ethics 2008; 34(10): 757-60. doi:10.1136/jme.2007. 022632

11 Kravitz RL, Duan N, White RH. N-of-1 trials of expensive biological therapies: a third way? Arch Intern Med 2008; 168(10): 1030-3. doi:10.1001/archinte.168.10.1030

12 Kravitz RL, Duan N, Niedzinski EJ, Hay MC, Subramanian SK, Weisner TS. What ever happened to N-of-1 trials? Insiders' perspectives and a look to the future. Milbank $Q 2008$; 86(4): 533-55. doi:10.1111/ j.1468-0009.2008.00533.x

13 Brookes ST, Biddle L, Paterson C, Woolhead G, Dieppe P. "Me's me and you's you": Exploring patients' perspectives of single patient (n-of-1) trials in the UK. Trials 2007; 8: 10. doi:10.1186/1745-6215-8-10

14 Tate RL, McDonald S, Perdices M, Togher L, Schultz R, Savage S. Rating the methodological quality of single-subject designs and n-of-1 trials: introducing the single-case experimental design (SCED) scale. Neuropsychol Rehabil 2008; 18(4): 385-401. doi:10.1080/ 09602010802009201

15 Smith $\mathrm{R}$. The ethics of $\mathrm{N}$ of 1 trials in routine practice and other problems of publication ethics. Cases $J$ 2008; 1(1): 78. doi:10.1186/ 1757-1626-1-78

Manuscript received 27 May 2008, accepted 1 April 2009 